\title{
Analysis of the Relationship between Ligamentum Flavum Thickening and Lumbar Segmental Instability, Disc Degeneration, and Facet Joint Osteoarthritis in Lumbar Spinal Stenosis
}

\author{
Toyomi Yoshiiwa, Masashi Miyazaki, Naoki Notani, \\ Toshinobu Ishihara, Masanori Kawano, Hiroshi Tsumura \\ Department of Orthopaedic Surgery, Oita University Faculty of Medicine, Yufu, Japan
}

\begin{abstract}
Study Design: Cross-sectional study.
Purpose: To investigate the relationship between ligamentum flavum (LF) thickening and lumbar segmental instability and disc degeneration and facet joint osteoarthritis.

Overview of Literature: Posterior spinal structures, including LF thickness, play a major role in lumbar spinal canal stenosis pathogenesis. The cause of LF thickening is multifactorial and includes activity level, age, and mechanical stress. LF thickening pathogenesis is unknown.

Methods: We examined 419 patients who underwent computed tomography (CT) myelography and magnetic resonance imaging after complaints of clinical symptoms. To investigate LF hypertrophy, 57 patients whose lumbar vertebra had normal disc heights at L4-5 were selected to exclude LF buckling as a hypertrophy component. LF thickness, disc space widening angulation in flexion, segmental angulation, presence of a vacuum phenomenon, and lumbar lordosis at T12-S1 were investigated. Disc and facet degeneration were also evaluated. Facet joint orientation was measured via an axial CT scan.

Results: The mean LF thickness in all patients was $4.4 \pm 1.0 \mathrm{~mm}$ at $L 4-5$. There was a significant correlation between $L F$ thickness and disc degeneration; LF thickness significantly increased with severe disc degeneration and facet joint osteoarthritis. There was a tendency toward increased LF thickness in more sagittalized facet joints than in coronalized facet joints. Logistic regression analysis showed that LF thickening was influenced by segmental angulation and facet joint osteoarthritis. Patient age was associated with LF thickening. Conclusions: LF hypertrophy development was associated with segmental instability and severe disc degeneration, severe facet joint osteoarthritis, and a sagittalized facet joint orientation.
\end{abstract}

Keywords: Disc degeneration; Ligamentum flavum thickness; Lumbar spine; Facet orientation

\section{Introduction}

Posterior spinal structures, including ligamentum flavum
(LF) thickness, play a major role in lumbar spinal canal stenosis (LSCS) pathogenesis [1-3]. The LF covers a considerable part of the posterior and lateral spinal canal

Received Mar 24, 2016; Revised May 23, 2016; Accepted Jun 8, 2016

Corresponding author: Masashi Miyazaki

Department of Orthopaedic Surgery, Oita University Faculty of Medicine,

1-1 Idaigaoka, Hsama-machi, Yufu, 879-5593, Japan

Tel: +81-97-586-5872, Fax: +81-97-586-6647, E-mail: masashim@med.oita-u.ac.jp 
walls [4]. As LF thickening can reduce spinal canal diameter, LF thickness has been considered as a cause of LSCS [5]. Canal narrowing mechanically compresses the nerve root or cauda equina [6-9] and contributes to lower back pain and sciatica, even in the absence of a bulging annulus fibrosus, herniated nucleus pulposus, or osseous spurs [10].

LF thickening pathogenesis is unclear, and whether the process is attributable to tissue hypertrophy or buckling remains controversial. Canal narrowing, in part, results from LF hypertrophy. In a normal ligament, elastic fibers are predominant and comprise $60 \%$ to $70 \%$ of the extracellular matrix $[11,12]$. In contrast, a hypertrophied LF shows an increased amount of collagen fibers, calcification, ossification, and chondrometaplasia [13-18].

The cause of LF hypertrophy is multifactorial and includes activity level, age, and mechanical stress. LF thickness in LSCS without disc space narrowing may sometimes be detected by radiographic findings. In such cases, LF thickness is not caused by LF buckling, but by LF hypertrophy. Mechanical stress-induced tissue damage might be the triggering event for an inflammatory reaction and subsequent development of tissue scarring $[16,19]$. The accumulation of such scarring may result in LF hypertrophy [20]. This study aimed to clarify LF hypertrophy pathogenesis associated with mechanical stress by investigating the relationship between segmental instability, disc degeneration, and facet joint osteoarthritis.

\section{Materials and Methods}

\section{Participants}

Prospective participants included 419 patients who underwent computed tomography (CT) myelography and magnetic resonance imaging (MRI) after complaining of clinical symptoms related to the spine, spinal cord, or cauda equina. Patients were evaluated between January 2007 and September 2013. Patients with evidence of bone metastasis, congenital anomalies, scoliosis, discitis, osteomyelitis, spondylolysis, spondylolisthesis, ossification, or fracture were excluded. Furthermore, with the intended purpose of investigating pure LF hypertrophy, 57 patients (13.6\%) with a normal L4-L5 disc height were selected. A previous study reported that the disc height for healthy males aged 20 to 30 years was $11.8 \mathrm{~mm}$ [21]. Therefore, we selected patients whose disc height was more than two- thirds $(8 \mathrm{~mm})$ the normal measurement. The study group comprised 41 men and 16 women with an average age of 63.2 years (range, $22-89$ years).

\section{LF thickness measurement}

Lumbar images were obtained using a 1.5 Tesla magnet (EXCELART Vantage, Toshiba Medical, Tokyo, Japan). LF thickness was measured at L4-5 for 57 patients on axial T2-weighted MRI images at the facet joint level (Fig. 1). Thickness at the middle LF portion was measured. Images were projected using a DICOM viewer. LF thickness was measured using electronic calipers at a DICOM workstation. Measurements were repeated twice by the same orthopedic surgeon; mean values were recorded. In asymmetrical thickness cases, the larger LF thickness value was used.

\section{Assessment of lumbar segmental spinal motion}

A radiologic assessment of lumbar segmental spinal motion was measured on dynamic film at flexion-extension. Disc space widening angulation in flexion and segmental angulation (angle of extension-angle of flexion) on lateral radiographs of extension and flexion were used to assess radiologic instability at the L4-5 segment. The presence of a vacuum phenomenon in the L4-5 disc and lumbar lordosis at T12-S1 was investigated by lateral radiograph.

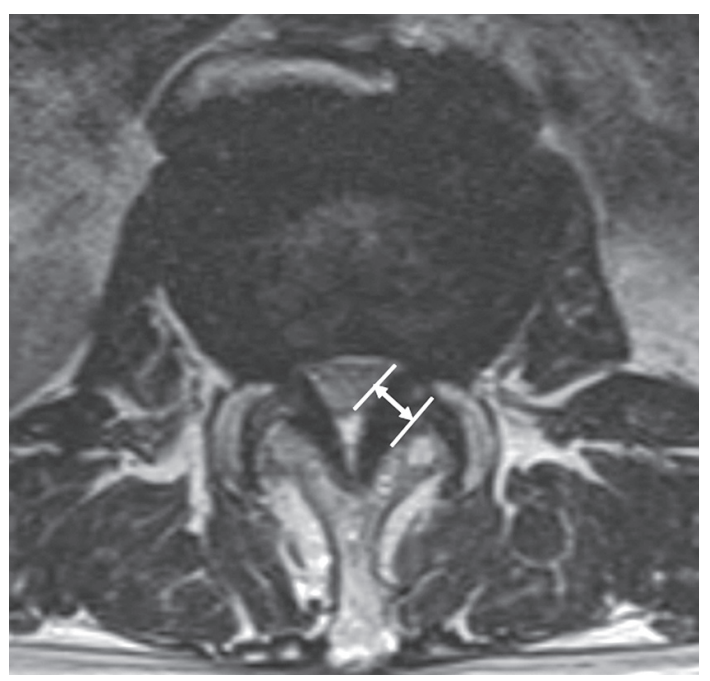

Fig. 1. Measurement of ligamentum flavum thickness on axial T2-weighted images at the facet joint level. 


\section{Disc degeneration assessment}

To evaluate vertebral discs, a major component for segmental stability, disc degeneration was classified into five grades according to Pfirmann's criteria using T2-weighted sagittal images [22]. The grade was determined by the degenerative status of the nucleus, annulus, and endplate (grade I, normal; grade V, most advanced degeneration).

\section{Lumbar facet joint assessment}

Facet joint orientation was measured on an axial CT scan. A facet line was drawn between the anteromedial and posterolateral points of the L4-5 facet. A mid-sagittal line, drawn through the disc, was considered the sagittal line. The angle formed by the left or right facet line and mid-

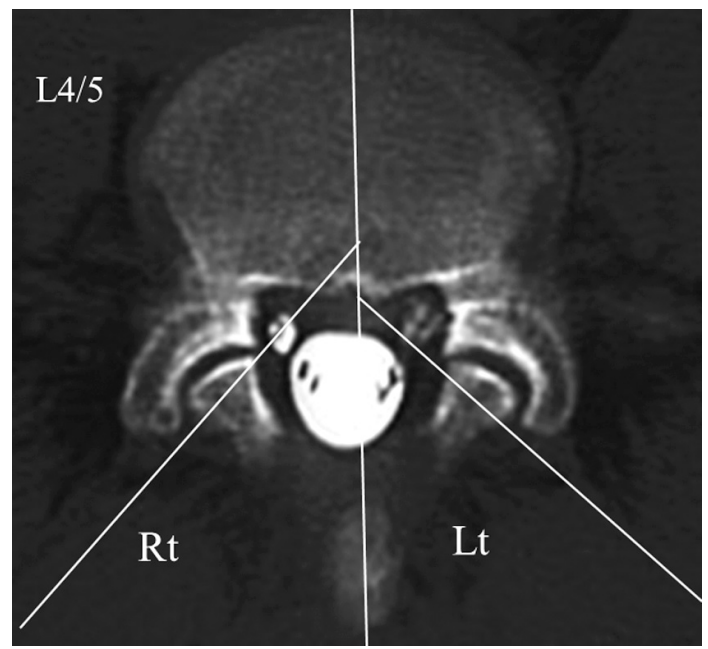

Fig. 2. Measurement of the facet angle on an axial computed tomography scan at the facet joint level.
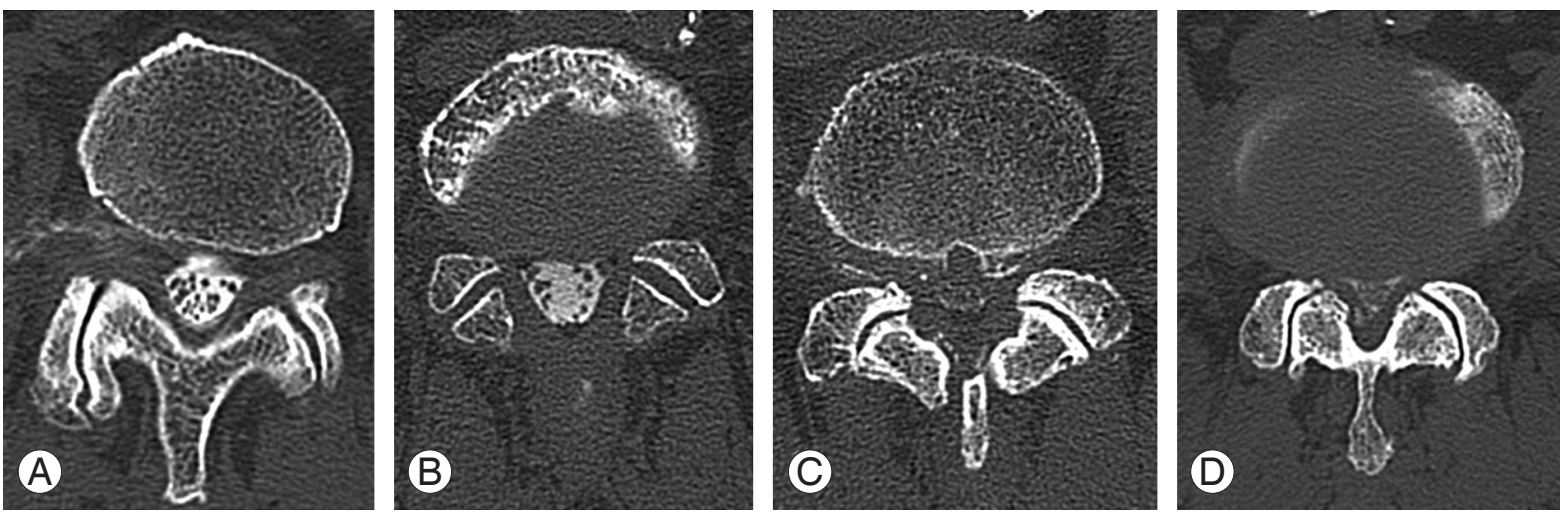

Fig. 3. The morphology of facet joints was classified into four groups. (A) sagittalized facet indicating a smaller facet angle. (B) Coronalized facet (angle of nearly $90^{\circ}$ ). (C) C-shaped facet (facet angle of nearly $45^{\circ}$ ). (D) J-shaped facet (facet angle of nearly $30^{\circ}$ ). sagittal line was measured and recorded in degrees (Fig. 2). Two angles (left and right) were averaged and recorded as facet joint orientation.

Facet joint morphology was classified into four groups according to Imajo et al. [23]: sagittalized facet joints indicated a smaller facet angle, coronalized facet joints were nearly $90^{\circ}, \mathrm{C}$-shaped facet joints were angled at about $45^{\circ}$, and J-shaped facet joints were angled at about $30^{\circ}$ (Fig. 3 ).

To evaluate facet joint osteoarthritis, osteophyte size at the L4-L5 facet joints was determined and classified into four grades according to Grogan et al. [24]: Grade 1 indicates no osteophyte; grade 2 , a mild or possible osteophyte; grade 3, a moderate osteophyte; and grade 4, a large osteophyte. When different grades were assigned to the right and left facet joints in the same segment, the grade for the most severe osteoarthritis was used.

\section{Statistical analysis}

Statistical analyses were performed with SPSS ver. 18.0 (SPSS Inc., Chicago, IL, USA). Relationships between LF thickness and age, disc widening angulation, segmental angulation, a vacuum phenomenon, lumbar lordosis, facet angle, disc degeneration, and facet joint osteoarthritis were analyzed by Spearman's correlation. Bonferroni correction was used to examine the relationship between $\mathrm{LF}$ thickness and disc degeneration, facet joint osteoarthritis, and facet joint orientation. A logistic regression analysis was performed to test whether each parameter was independently associated with LF thickness. Studies have proposed to set an upper limit for normal LF thickness of $<4$ $\mathrm{mm}[25,26]$. Therefore, LF thickening was identified when LF thickness was $\geq 4 \mathrm{~mm}$. Logistic regression was applied 
Table 1. Relationships between thickness of LF and parameters

\begin{tabular}{lcc} 
Parameter & Value & $p$-value \\
Age $(y r)$ & 63.2 & $S(p<0.01, r=0.55)$ \\
Angulation of disc space widening $\left(^{\circ}\right)$ & $4.2 \pm 5.2$ & NS $(p=0.82, r=-0.31)$ \\
\hline Segmental angulation $\left({ }^{\circ}\right)$ & $6.8 \pm 3.4$ & $N S(p=0.60, r=0.07)$ \\
Lumbar lordosis $\left(^{\circ}\right)$ & $45.2 \pm 12.1$ & NS $(p=0.90, r=-0.17)$ \\
Vacuum phenomenon & $14 / 57(24.6 \%)$ & $S(p<0.05, r=0.32)$ \\
Facet angle $\left(^{\circ}\right)$ & $40.9 \pm 8.4$ & $N S(p=0.35, r=-0.13)$ \\
\hline
\end{tabular}

The values are given as the mean and the standard deviation.

LF, ligamentum flavum; S, significant; $r$, correlation coefficient; NS, not significant.

to determine which variables could predict the risk of LF thickness, with the end point being $\geq 4 \mathrm{~mm}$. Odds ratios (ORs) and 95\% confidence intervals were determined for each predictive variable. Demographics and radiological parameters according to an LF of $<4 \mathrm{~mm}$ or $\geq 4 \mathrm{~mm}$ were statistically analyzed by the Mann-Whitney $U$ test. The grade system of disc degeneration and facet joint osteoarthritis was compared at $<4 \mathrm{~mm}$ and $\geq 4 \mathrm{~mm}$ by the chisquare test. Statistical significance was defined as $p<0.05$. Intraobserver and interobserver agreement were assessed to be good-to-excellent with a kappa statistic of $>0.70$ for each parameter.

\section{Results}

\section{Lumbar segmental spinal motion, disc degeneration, and facet joint orientation}

The mean disc space widening angulation at L4-L5 was $4.2^{\circ} \pm 5.2^{\circ}$ (range, $-10^{\circ}$ to $+17^{\circ}$ ). The mean segmental angulation at L4-L5 was $6.8^{\circ} \pm 3.4^{\circ}$ (range, $0^{\circ}$ to $+15^{\circ}$ ). The average lumbar lordosis was $45.2^{\circ} \pm 12.1^{\circ}$ (range, $+15^{\circ}$ to $\left.+76^{\circ}\right)$. Fourteen patients $(24.6 \%)$ had a vacuum phenomenon (Table 1). Disc degeneration was identified as grade II in 4 patients (7.0\%), grade III in 20 (35.1\%), and grade IV in $33(57.9 \%)$. No patients had grade I or V.

The mean facet angle was $40.9^{\circ} \pm 8.4^{\circ}$ (range, $23.5^{\circ}$ to $55.5^{\circ}$ ), and facet joint morphology was sagittalized in 2 patients (3.5\%), coronalized in 3 (5.2\%), C-shaped in 36 (63.2\%), and J-shaped in 16 (28.1\%). Facet joint osteoarthritis was grade 1 in 1 patient (1.8\%), grade 2 in 19 (33.3\%), grade 3 in 28 (49.1\%), and grade 4 in 9 (15.8\%). The Bonferroni correction was not valid because the grade 1 group included only one patient.

\section{Relationships between LF thickness and parameters}

The mean LF thickness was $4.4 \pm 1.0 \mathrm{~mm}$ for L4-L5. LF thickness significantly correlated with age $(r=0.55, p<0.01)$ and the vacuum phenomenon $(r=0.32, p<0.05)$ and was independent of disc space widening angulation, segmental angulation, lumbar lordosis, and facet angle $(p>0.05)$ (Table 1).

The grade distribution of disc degeneration was as follows: grade II, was $3.2 \pm 0.1 \mathrm{~mm}$; grade III, was $3.9 \pm 0.7$ $\mathrm{mm}$; and grade IV, was $4.8 \pm 1.0 \mathrm{~mm}$. LF thickness was greater in the grade IV group compared withthan in the grade II $(p<0.01)$ and grade III $(p<0.01)$ groups, with a significant positive association between LF thickness and

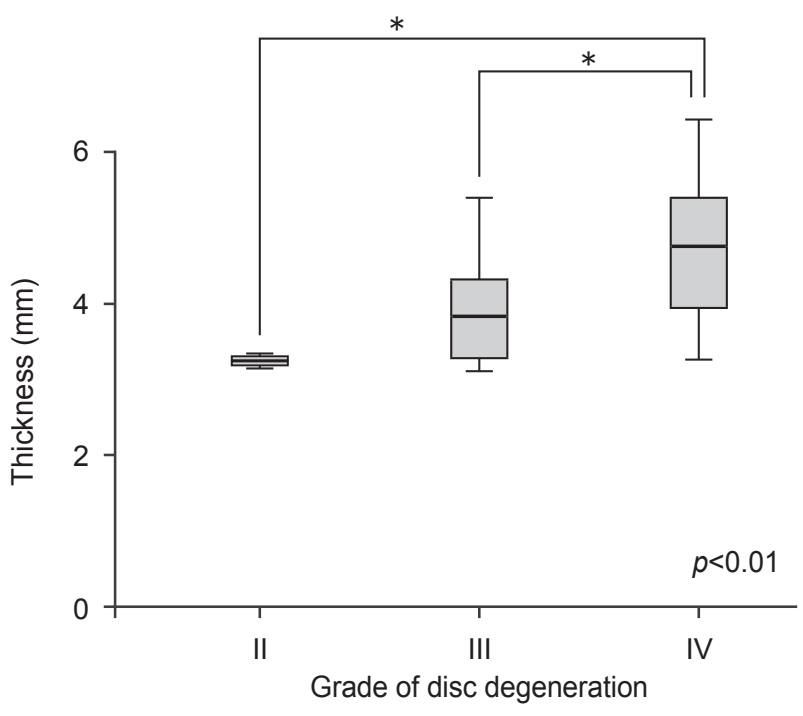

Fig. 4. Mean ligamentum flavum (LF) thickness of each grade of disc degeneration. The average thickness of grade II was $3.2 \pm 0.1 \mathrm{~mm}$, grade III was $3.9 \pm 0.7 \mathrm{~mm}$, and grade IV was $4.8 \pm 1.0 \mathrm{~mm}$. LF thickness was greater in grade IV than in grade II $(p<0.01)$ and grade III $(p<0.01)$. 
increasing disc degeneration severity (Fig. 4). In addition, LF thickness was significantly correlated with age ( $r=0.55$, $p<0.01)$. Furthermore, age was significantly correlated with disc degeneration $(r=0.67, p<0.01)$ and facet joint osteoarthritis $(r=0.56, p<0.01)$ (Fig. 5). This association between LF thickness and age was mirrored by a significant positive correlation between age and grade of disc degeneration and facet joint osteoarthritis.

Regarding facet joint morphology, the average sagittalized facet thickness was $5.0 \pm 1.8 \mathrm{~mm}$; coronalized facet thickness was $3.9 \pm 0.8 \mathrm{~mm}, \mathrm{C}$-shaped facet thickness was $4.4 \pm 1.0 \mathrm{~mm}$, and J-shaped facet thickness was $4.3 \pm 0.8$ $\mathrm{mm}$ (Fig. 6). No statistical significance was noted, but LF thickness was greater for more sagittalized facet joints than more coronalized facet joints.

Thirty-one patients (54.4\%) had an LF thickness of $\geq 4 \mathrm{~mm}$ and $26(45.6 \%)$ had an LF thickness of $<4 \mathrm{~mm}$. Table 2 shows demographics and radiological parameters according to an LF thickness of $<4 \mathrm{~mm}$ or $\geq 4 \mathrm{~mm}$. Logistic regression analysis showed that $\mathrm{LF}$ thickening was influenced by disc space widening (OR, $0.86 ; p=0.033)$ and facet joint osteoarthritis (OR, 3.57; $p=0.032)$. Patient age was also associated with LF thickening (OR, 1.08; $p=0.041$ ) (Table 3). Conversely, segmental angulation, the vacuum phenomenon, and lumbar lordosis were not associated with LF thickening. Disc degeneration and facet joint osteoarthritis were the result of aging, and thus lumbar segmental instability occurred because of lumbar disc

The thickness of LF in each facets shape

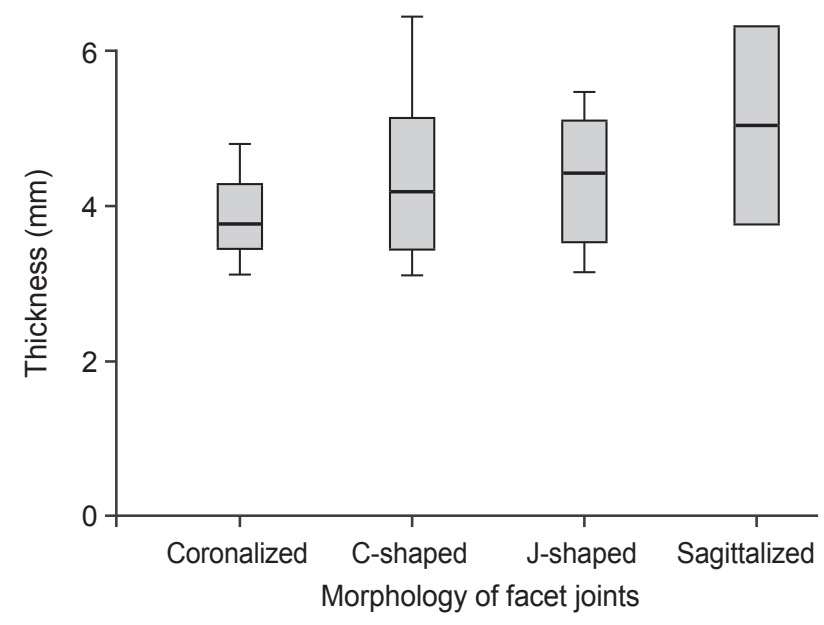

Fig. 6. The average thickness in the four groups. LF, ligamentum flavum.

Relationship between age and both disc degeneration and facet joints osteoarthritis
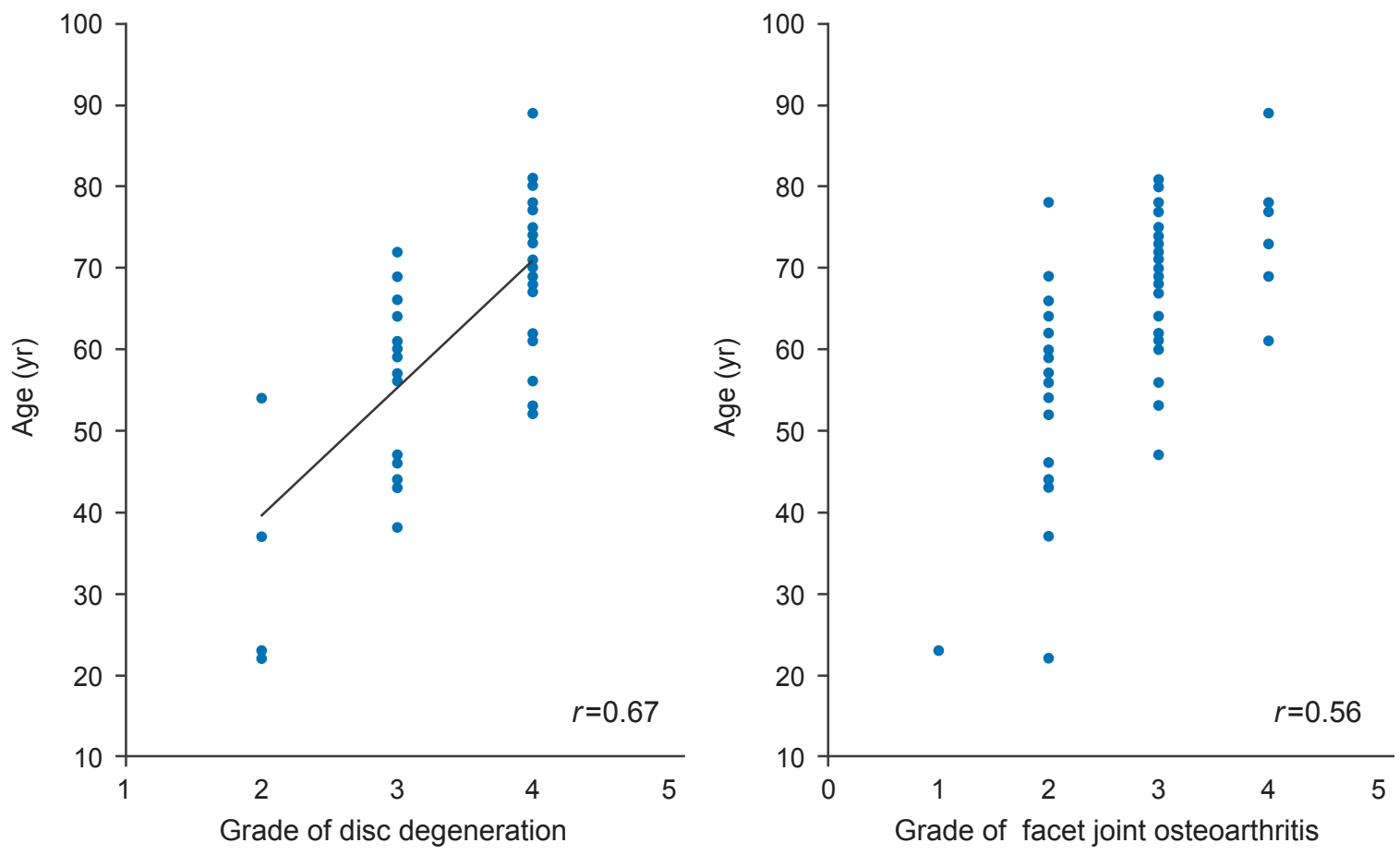

Fig. 5. Age significantly correlated with disc degeneration $(r=0.67, p<0.01)$ and facet joint osteoarthritis $(r=0.56, p<0.01)$. 
Table 2. Measured parameters of $<4 \mathrm{~mm}$ and $\geq 4 \mathrm{~mm}$ LF thickness

\begin{tabular}{|c|c|c|c|}
\hline Parameter & $<4 \mathrm{~mm}$ & $\geq 4 \mathrm{~mm}$ & $p$-value \\
\hline Age $(y r)$ & $56.3 \pm 2.9$ & $68.9 \pm 1.7$ & $S(p<0.01)$ \\
\hline Angulation of disc space widening $\left({ }^{\circ}\right)$ & $5.4 \pm 0.8$ & $3.2 \pm 1.0$ & NS $(p=0.14)$ \\
\hline Segmental angulation $\left(^{\circ}\right)$ & $6.4 \pm 0.7$ & $7.1 \pm 0.6$ & NS $(p=0.38)$ \\
\hline Lumbar lordosis $\left({ }^{\circ}\right)$ & $45.6 \pm 2.6$ & $44.9 \pm 2.0$ & NS $(p=0.60)$ \\
\hline Vacuum phenomenon, n (\%) & $2 / 57(4.0)$ & $12 / 57(21.1)$ & $S(p<0.01)$ \\
\hline \multicolumn{4}{|l|}{ Disc degeneration } \\
\hline Grade I and V & $0 / 26$ & $0 / 31$ & NA \\
\hline Grade II & $4 / 26$ & $0 / 31$ & $S(p<0.05)$ \\
\hline Grade III & $13 / 26$ & $7 / 31$ & $S(p<0.05)$ \\
\hline Grade IV & $9 / 26$ & $24 / 31$ & $S(p<0.05)$ \\
\hline Facet angle $\left({ }^{\circ}\right)$ & $41.8 \pm 0.1$ & $40.2 \pm 0.1$ & NS $(p=0.28)$ \\
\hline \multicolumn{4}{|l|}{ Facet joint osteoarthritis } \\
\hline Grade 1 & $1 / 26$ & $0 / 31$ & NS $(p=0.27)$ \\
\hline Grade 2 & $15 / 26$ & $4 / 31$ & $S(p<0.01)$ \\
\hline Grade 3 & $8 / 26$ & $20 / 31$ & NS $(p=0.07)$ \\
\hline Grade 4 & $2 / 26$ & $7 / 31$ & NS $(p=0.15)$ \\
\hline
\end{tabular}

The values are given as the mean and the standard deviation. Statistical analyses were performed by Mann-Whitney U test. The grade system of disc degeneration and facet joint osteoarthritis were by chi-square test.

LF, ligamentum flavum; S, significant; NS, not significant; NA, not available.

Table 3. Logistic regression analysis for the thickness of LF

\begin{tabular}{lccc} 
Independent variable & $p$-value & OR & $95 \% \mathrm{Cl}$ \\
Age & 0.041 & 1.08 & $1.00-1.15$ \\
Angulation of disc space widening & 0.033 & 0.86 & $0.75-0.99$ \\
\hline Facet joints osteoarthritis & 0.032 & 3.57 & $1.11-11.44$ \\
\hline
\end{tabular}

LF, ligamentum flavum; $\mathrm{OR}$, odds ratio; $\mathrm{Cl}$, confidence interval.

space widening and sagittalized facet joints.

\section{Discussion}

The LF covers the posterior spinal canal wall. LF hypertrophy may eventually compress the spinal cord, cauda equina, or nerve roots [6,27-29]. Canal narrowing in LSCS results from degenerative changes in posterior lumbar spine structures, with LF thickening and bony proliferation of the facet joints being major factors in its development [19]. The mechanism of LF hypertrophy from the perspectives of anatomy, histology, and biology has been investigated. Causes of LF hypertrophy include age, spinal level, mechanical stress endured, and growth factors. Histological research data have shown that an increased LF thickness is associated with an increase in fibrosis (i.e., an increase in collagen fiber content) and a decrease in elastic fibers [13,16-18]. Epidemiologically, mechanical stress attributable to spinal instability may initiate LF hypertrophy $[16,25,30,31]$. However, the mechanism inciting lumbar mechanical stress remains unknown. In our study, we used radiographic measures of L4-5 segmental instability to elucidate the mechanism between mechanical stress and LF thickness.

LF thickening pathogenesis is unclear, and whether LF thickening is due to tissue hypertrophy or buckling remains controversial. The exact function of the LF is also unknown, but it is a connective tissue thought to affect intrinsic spine stability, control intervertebral movement, and maintain a smooth posterior dural sac surface [32]. Using a transmission electron microscopy LF assessment, Postacchini et al. [14] concluded that reduced elasticity 
may cause the LF to bulge into the spinal canal, even in the standing position with an LF of normal thickness. In addition, a cause of thickening may be LF buckling into the spinal canal after disc collapse. To evaluate this theory, Altinkaya et al. [33] examined the relationship between LF thickness and intervertebral disc degeneration and disc height; they reported a significant increase in LF thickness with increased disc degeneration. Based on these findings, they hypothesized that spinal segment buckling, secondary to disc degeneration, resulted in LF thickening and buckling into the spinal canal. Conversely, Sakamaki et al. [4] did not identify a correlation between LF thickness and disc degeneration. We investigated patients with preserved disc height because each intervertebral disc has a different segmental mobility. During flexion-extension, there is usually a cephalocaudal increase in the range of motion in the lumbar spine; the L4-5 area bears the highest load and tends to experience the most motion. Our study included 57 patients (13.6\%) with preserved L4-5 disc heights; 31 (7.4\%) patients had an LF thickness of $\geq 4$ $\mathrm{mm}$, LF hypertrophy, and no buckling. Furthermore, disc degeneration and facet joint osteoarthritis significantly correlated with aging; LF thickness also significantly correlated with disc degeneration and facet joints osteoarthritis. In our study, LF thickness increased with age, supporting previous research suggesting that LF thickness is an age-dependent phenomenon $[4,16,34]$. Thus, our results indicate that thickening is not necessarily LF buckling into the spinal canal associated with disc degeneration.

Normal LF is composed of $80 \%$ elastic fibers and 20\% collagen fibers [35]. Upon hypertrophy, LF shows a loss of elastic fibers and an increase in collagen fibers, resulting in fibrosis $[17,20]$. Previous histological data indicated that LF hypertrophy was characterized by fibrosis, loss of elastic fibers, increased collagen fibers, calcification, ossification, degeneration of elastic fibers, and chondrometaplasia [13,16,18-20]. These histological changes are similar to tissue scarring during the post-inflammatory repair process in other organs [36-39]. Sairyo et al. [16,19] reported that damaged LF tissue, induced by mechanical stress, might be the initial trigger for an inflammatory reaction and subsequent development of tissue scarring. Attention has recently focused on angiogenesis, a pivotal component of scarring and the tissue repair process. Moon et al. [40] reported that LF cells interact with macrophage-like cells to produce angiogenesis-related factors, with the exception of transforming growth factor $\beta 1$. Activated LF cells exposed to macrophages can impact the stimulus of angiogenesis-related factors. This indicates that fibrosis and scarring, triggered by an inflammatory reaction, is the major pathomechanism of LF hypertrophy. Therefore, inflammatory reactions are initiated by trauma such as mechanical stress and are followed by the repair process. Hypertrophied LF, containing more collagen fibers, is vulnerable to repetitive flexion-extension motion resulting in a vicious cycle of scar-repair-scar.

Mechanical stress is one of the most important factors in LF hypertrophy, yet it is unclear precisely what form of mechanical stress induces hypertrophy. Fukuyama et al. [25] reported that LF with radiological degenerative signs such as spondylolisthesis or a vacuum phenomenon exhibits severe hypertrophy and suggested that mechanical instability is a possible cause of LF hypertrophy. In our study, disc space widening was identified as a significant risk factor of LF thickening, although the vacuum phenomenon was not retained as a predictive factor in our logistic regression analysis. Abbas et al. [34] reported that LF thickness in the LSCS group occurred at L3-4 and L4-5 segments because of the relative hypermobility of these two segments compared with the L5-S1 segment, which is stabilized by the iliolumbar ligaments and the large transverse processes of the L5 vertebra. Sairyo et al. [16] investigated LF thickness and used a three-dimensional finite element model to characterize the effects of mechanical stresses on various LF layers. Maximum stress was observed in flexion mode activities such as lifting. More biomechanical stress on the dorsal side of the LF than the ventral side led to LF hypertrophy.

In our study, a logistic regression analysis showed that LF thickening was influenced by disc space widening, facet joint osteoarthritis, and patient age. The possibility of a relationship between LF thickening and facet joint osteoarthritis has rarely been reported. Wang and Yang [41] suggested that a coronal L4-5 facet joint orientation negatively correlated to age, which could explain why aging people are prone to degenerative spondylolisthesis; degenerative spondylolisthesis is considered to be one of the causes of induced mechanical stress. In our study, a greater LF thickness was associated with a more sagittalized facet joint orientation and facet joint osteoarthritis. These findings suggest that the aging process increases disc degeneration and affects the facet joints, possibly leading to segmental instability on flexion-extension motion. Therefore, repetitive flexion-extension motion cycles 
may lead to LF hypertrophy.

Our study has several limitations. Foremost, numerous factors contribute to the narrowing of the spinal canal beyond LF thickening, including developmental canal stenosis or spondylolisthesis. To further understand canal narrowing pathogenesis, these additional factors should be included in future analyses. Second, our study group was small and did not include a control group. Therefore, the correlation between LF thickness and LSCS symptoms could not be evaluated within the context of our study. In addition, LF thickening was defined by a thickness of $\geq 4 \mathrm{~mm}$, representing a narrow range in comparison with previous studies $[16,25,26,34,42,43]$.

\section{Conclusions}

LF thickness correlated with age, disc space widening angulation, and facet joint osteoarthritis. LF hypertrophy development was associated with segmental instability and severe disc degeneration, severe facet joints osteoarthritis, and sagittalized facet joint orientation.

\section{Conflict of Interest}

No potential conflict of interest relevant to this article was reported.

\section{References}

1. Grenier N, Kressel HY, Schiebler ML, Grossman RI, Dalinka MK. Normal and degenerative posterior spinal structures: MR imaging. Radiology 1987;165:51725.

2. Schneck CD. The anatomy of lumbar spondylosis. Clin Orthop Relat Res 1985;(193):20-37.

3. Tsuji H, Itoh T, Tamaki T. Natural history and pathogenesis of the lumbar spinal stenosis. Rinsho Seikei Geka 1981;16:598-612.

4. Sakamaki T, Sairyo K, Sakai T, Tamura T, Okada Y, Mikami H. Measurements of ligamentum flavum thickening at lumbar spine using MRI. Arch Orthop Trauma Surg 2009;129:1415-9.

5. Elsberg CA. Experiences in spinal surgery: observation upon 60 laminectomies for spinal diseases. Surg Gynecol Obset 1913;16:117-35.

6. Beamer YB, Garner JT, Shelden CH. Hypertrophied ligamentum flavum: clinical and surgical signifi- cance. Arch Surg 1973;106:289-92.

7. Brown HA. Enlargement of the ligamentum flavum. J Bone Joint Surg Am 1938;20:325-38.

8. Rauschning W. Normal and pathologic anatomy of the lumbar root canals. Spine (Phila Pa 1976) 1987; 12:1008-19.

9. Schonstrom NS, Bolender NF, Spengler DM. The pathomorphology of spinal stenosis as seen on CT scans of the lumbar spine. Spine (Phila Pa 1976) 1985; 10:806-11.

10. Kirkaldy-Willis WH. The relationship of structural pathology to the nerve root. Spine (Phila Pa 1976) 1984;9:49-52.

11. Evans JH, Nachemson AL. Biomechanical study of human lumbar ligamentum flavum. J Anat 1969;105: 188-9.

12. Nachemson AL, Evans JH. Some mechanical properties of the third human lumbar interlaminar ligament (ligamentum flavum). J Biomech 1968;1:211-20.

13. Okuda T, Baba I, Fujimoto Y, et al. The pathology of ligamentum flavum in degenerative lumbar disease. Spine (Phila Pa 1976) 2004;29:1689-97.

14. Postacchini F, Gumina S, Cinotti G, Perugia D, DeMartino C. Ligamenta flava in lumbar disc herniation and spinal stenosis.: light and electron microscopic morphology. Spine (Phila Pa 1976) 1994;19: 917-22.

15. Ramsey RH. The anatomy of the ligamenta flava. Clin Orthop Relat Res 1966;44:129-40.

16. Sairyo K, Biyani A, Goel V, et al. Pathomechanism of ligamentum flavum hypertrophy: a multidisciplinary investigation based on clinical, biomechanical, histologic, and biologic assessments. Spine (Phila Pa 1976) 2005;30:2649-56.

17. Schrader PK, Grob D, Rahn BA, Cordey J, Dvorak J. Histology of the ligamentum flavum in patients with degenerative lumbar spinal stenosis. Eur Spine J 1999;8:323-8.

18. Yoshida M, Shima K, Taniguchi Y, Tamaki T, Tanaka T. Hypertrophied ligamentum flavum in lumbar spinal canal stenosis. Pathogenesis and morphologic and immunohistochemical observation. Spine (Phila Pa 1976) 1992;17:1353-60.

19. Sairyo K, Biyani A, Goel VK, et al. Lumbar ligamentum flavum hypertrophy is due to accumulation of inflammation-related scar tissue. Spine (Phila Pa 1976) 2007;32:E340-7. 
20. Kosaka H, Sairyo K, Biyani A, et al. Pathomechanism of loss of elasticity and hypertrophy of lumbar ligamentum flavum in elderly patients with lumbar spinal canal stenosis. Spine (Phila Pa 1976) 2007;32: 2805-11.

21. Roberts N, Gratin C, Whitehouse GH. MRI analysis of lumbar intervertebral disc height in young and older populations. J Magn Reson Imaging 1997;7: 880-6.

22. Pfirrmann CW, Metzdorf A, Zanetti M, Hodler J, Boos N. Magnetic resonance classification of lumbar intervertebral disc degeneration. Spine (Phila Pa 1976) 2001;26:1873-8.

23. Imajo Y, Kato Y, Taguchi T. Facet joint morphology of degenerative spondylolisthesis at L4-5. Spine Spinal Cord 2012;25:315-20.

24. Grogan J, Nowicki BH, Schmidt TA, Haughton VM. Lumbar facet joint tropism does not accelerate degeneration of the facet joints. AJNR Am J Neuroradiol 1997;18:1325-9.

25. Fukuyama S, Nakamura T, Ikeda T, Takagi K. The effect of mechanical stress on hypertrophy of the lumbar ligamentum flavum. J Spinal Disord 1995;8:12630.

26. Park JB, Chang H, Lee JK. Quantitative analysis of transforming growth factor-beta 1 in ligamentum flavum of lumbar spinal stenosis and disc herniation. Spine (Phila Pa 1976) 2001;26:E492-5.

27. Towne EB, Reichert FL. Compression of the lumbosacral roots of the spinal cord by thickened ligamenta flava. Ann Surg 1931;94:327-36.

28. Naylor A. Factors in the development of the spinal stenosis syndrome. J Bone Joint Surg Br 1979;61:3069.

29. Young-Hing K, Reilly J, Kirkaldy-Willis WH. The ligamnetum flavum. Spine 1976;1:226-34.

30. Nakamura T, Hashimoto N, Maeda Y, Ikeda T, Nakagawa $\mathrm{H}$, Takagi K. Degeneration and ossification of the yellow ligament in unstable spine. J Spinal Disord 1990;3:288-92.

31. Nakatani T, Marui T, Hitora T, Doita M, Nishida K, Kurosaka M. Mechanical stretching force promotes collagen synthesis by cultured cells from human ligamentum flavum via transforming growth factorbeta1. J Orthop Res 2002;20:1380-6.
32. Safak AA, Is M, Sevinc O, et al. The thickness of the ligamentum flavum in relation to age and gender. Clin Anat 2010;23:79-83.

33. Altinkaya N, Yildirim T, Demir S, Alkan O, Sarica FB. Factors associated with the thickness of the ligamentum flavum: is ligamentum flavum thickening due to hypertrophy or buckling? Spine (Phila Pa 1976) 2011;36:E1093-7.

34. Abbas J, Hamoud K, Masharawi YM, et al. Ligamentum flavum thickness in normal and stenotic lumbar spines. Spine (Phila Pa 1976) 2010;35:1225-30.

35. Viejo-Fuertes D, Liguoro D, Rivel J, Midy D, Guerin J. Morphologic and histologic study of the ligamentum flavum in the thoraco-lumbar region. Surg Radiol Anat 1998;20:171-6.

36. Frangogiannis NG. Chemokines in the ischemic myocardium: from inflammation to fibrosis. Inflamm Res 2004;53:585-95.

37. Furuichi K, Kaneko S, Wada T. Chemokine/chemokine receptor-mediated inflammation regulates pathologic changes from acute kidney injury to chronic kidney disease. Clin Exp Nephrol 2009;13:914.

38. Strieter RM, Mehrad B. New mechanisms of pulmonary fibrosis. Chest 2009;136:1364-70.

39. Wilgus TA, Vodovotz Y, Vittadini E, Clubbs EA, Oberyszyn TM. Reduction of scar formation in fullthickness wounds with topical celecoxib treatment. Wound Repair Regen 2003;11:25-34.

40. Moon HJ, Park YK, Ryu Y, Kim JH, Kwon TH, Chung HS. The angiogenic capacity from ligamentum flavum subsequent to inflammation: a critical component of the pathomechanism of hypertrophy. Spine (Phila Pa 1976) 2012;37:E147-55.

41. Wang J, Yang X. Age-related changes in the orientation of lumbar facet joints. Spine (Phila Pa 1976) 2009;34:E596-8.

42. Park JB, Lee JK, Park SJ, Riew KD. Hypertrophy of ligamentum flavum in lumbar spinal stenosis associated with increased proteinase inhibitor concentration. J Bone Joint Surg Am 2005;87:2750-7.

43. Spurling RG, Mayfield FH, Rogers JB. Hypertrophy of the ligamenta flava as a cause of low back pain. JAMA 1937;109:928-33. 